\title{
CHEMOSPHERE
}

\section{Effects of polyelectrolytes on reduction of model compounds via coagulation}

\author{
E.-E. Chang ${ }^{a}$, Pen-Chi Chiang ${ }^{b, *}$, Wei-Yan Tang ${ }^{b}$, \\ Su-Hei Chao ${ }^{\text {a }}$, Hao-Jan Hsing ${ }^{b}$ \\ a Department of Biochemistry, Taipei Medical University, Taipei, Taiwan, ROC \\ ${ }^{\mathrm{b}}$ Graduate Institute of Environmental Engineering, National Taiwan University, \\ 71, Chou-Shan Road, Taipei, Taiwan 106, Taiwan, ROC
}

Received 17 March 2004

\begin{abstract}
The objective of this research work was to evaluate the performance of enhanced coagulation by alum and polymer. Synthetic source waters containing high molecular weight humic acids, medium molecular weight tannic acids and low molecular weight $p$-hydroxybenzoic acid were formulated by adjusting the concentration of turbidity and $\mathrm{pH}$; and jar tests were used to study the effect of various types and dosages of polymer on reducing the above model compounds.

At a specific $\mathrm{pH}$ condition, the applied alum dosage would efficiently decrease the turbidity to 2 NTU follows the order: humic $>$ tannic $>p$-hydroxybenzoic acid. Adjustment of $\mathrm{pH}$ influenced the performance of alum obviously but not of $p$-DADMAC. High $p$-DADMAC dosage overwhelming the effects of alum is less affected by $\mathrm{pH}$ adjustment.

The results of this investigation reveal that enhanced coagulation with $p$-DADMAC was founded to be very effective for removing high-molecular-weight THM precursors, i.e., humic acid and tannic acid, and markedly reduced the alum dosages required for turbidity removal. The other two polymers, i.e., cationic PAM and non-ionic PAM, which had higher molecular weight but lower charge density than $p$-DADMAC, were not capable of removing organic precursors. It was thus concluded that enhanced coagulation with polymer, $p$-DADMAC, could be considered as a promising technique for removal of NOMs with hydrophobic and higher-molar-mass $(>1 \mathrm{~K})$ in water treatment plants.
\end{abstract}

(c) 2004 Elsevier Ltd. All rights reserved.

Keywords: Enhance coagulation; Polymer; Polyacryamide; $p$-diallyldimethyl ammonium chloride; $p$-hydroxybenzoic acid; Tannic acid; Humic acid; Trichloromethane (THM) formation

\section{Introduction}

The coagulation process is optimized primarily for the removal of turbidity. Although, nature organic matter

\footnotetext{
* Corresponding author. Tel.: +88622362 2510; fax: +8862 23661642.

E-mail address: pcchiang@ntu.edu.tw (P.-C. Chiang).
}

(NOM) is also removed by coagulation, the removal efficiency varied with the physical and chemical characteristics of the water as well as the operating conditions of the coagulation process (Ratnaweera et al., 1999). Unless the raw water has a low total organic carbon (DOC) concentration, coagulant dosages are determined by the content of NOM in raw water rather than by turbidity (O'Melia et al., 1999). Generally, the higher-molar-mass fraction of organic matter (OM) is readily removed by 
coagulation. The type of $\mathrm{OM}$ in raw water is also a factor affecting its removal by coagulation. Functional groups of OM influence the solubility of organic compounds; hydrophobic OM is easier to be removed than hydrophilic OM (Collins et al., 1986; White et al., 1997). Owen et al. (1993) indicated that a large percentage of disinfection by products (DBPs) was formed from the non-humic fraction of NOM. This fraction is generally more hydrophilic than humic substances and thus more difficult to remove by coagulation.

Polymers have particular advantages over inorganic coagulants for NOM removal. The performance is less $\mathrm{pH}$ dependent and there is a lower level of dissolved ions in the product water. Mallevialle et al. (1984) found that chlorination of polyacryamide (PAM) and acryamide monomers shows low reactivity, and generated a small amount of total organic halides (TOX) and trihalomethane (THM). Chang et al. (1999) found that the polydiallyldimethyl ammonium chloride ( $p$-DADMAC) not only effectively removed the turbidity but also reduced the formation of THM. In evaluating cationic polyelectrolytes for the removal of UV absorbers, addition of alum followed by cationic polymethacrylate, $p$-DADMAC or cationic PAM (CPAM) was found to be effective. A polymer with higher charge density (CD) is more effective in reducing UV absorbers than that with low CD. $p$-DADMAC is not considered to be toxic and accepted for use in treatment of municipal water supplies by USEPA. However, the USEPA acceptance is only by the specific name of the suppliers and not by generic type, the maximum dose for $p$-DADMAC is $10 \mathrm{mgl}^{-1}$ (AWWA, 1987). PAM is a high molecular weight organic polymer and solute in water easily, and can resist the attack from microbial (Seybold, 1994). Chronic environmental studies indicated that no adverse effects were discovered in workers exposed to PAM dust over a period of 5 years. It has also been known to be non-toxic to human animals, and fish (Anonymous, 1991).

A thorough understanding of the reaction mechanism is a necessary step in determining the proper type of polymer to be used for the coagulation process. The reaction of polymers with other chemicals such as disinfectants in the form of chlorine may adversely affect the success of the coagulation process. Therefore, the objectives of this paper were intended to investigate the effects of three organics acids i.e., humic acid (HA), tannic acid (TA) and $p$-hydroxybenzoic acid (PHBA) on coagulation performance and THM formation potential as well as to determine the most suitable polymer as a coagulant-aid in the coagulation process.

\section{Materials and methods}

Three model organic compounds with different molar masses and degrees of hydrophobicity were used to sim- ulate some of the wide range of organics found in NOM (Exell and Vanloon, 2000). HA represents fairly hydrophobic, high-molar-mass (molecular weight $(\mathrm{MW})=10$ to 100 thousands) natural compounds and is a negatively charged polyelectrolyte due to the dominance of carboxylic acid groups. A number of previous studies have utilized this material; it represents a good model humic substance (Chang et al., 2001; Mustafa and Walker, 2001). TA represents relatively hydrophilic compounds of medium molar mass $(\mathrm{MW}=1700)$, and PHBA $(\mathrm{MW}=138)$ represents small organic molecules found in nature. All of the model compounds containing carboxylic and phenolic groups. Jar tests with rapid mixing, followed by settling were conducted to evaluate the efficiencies of the coagulant and coagulant-aid in removing these compounds and reducing turbidity, as well as THM formation potential under various $\mathrm{pH}$ conditions.

\subsection{Polymers}

Two types of polyacryamide (SNF Co.) including non-ionic PAM of high MW ranging from 5 to 15 million, and CPAM of positively-charged, with $\mathrm{CD}<15 \%$ containing very high molecular weight (3 to 15 million) were used in this study as coagulant aid. Another cationic polymer, $p$-DADMAC, which has a high CD $(100 \%)$ and varying MW was also used in this study.

\subsection{Synthetic water}

Synthetic water was made up to resemble the alkalinity, turbidity, and OM (HA, TA, and PHBA) levels of natural water. In 11 of distilled water, sodium bicarbonate was added to produce on alkalinity of $100 \pm 10 \mathrm{mgl}^{-1}$ as $\mathrm{CaCO}_{3}$, and $0.662 \mathrm{mg}$ bentonite was added to obtain an approximate turbidity of 200 NTU. The DOC of the synthetic water prepared above was near $7 \mathrm{mgl}^{-1}$ as $\mathrm{C}$. This solution was mixed on a stir plate for $1 \mathrm{~h}$ before being transferred to 211 . The water was then left in a closed container overnight $(>18 \mathrm{~h})$ and the $\mathrm{pH}$ was adjusted before it was used in jar tests.

\subsection{Jar tests and analyses}

All three coagulant-aids and each type of organic compounds were used to compare the effectiveness of each coagulant-aid in removing various types of OM and turbidity under different $\mathrm{pH}$ condition. The alum used as a coagulant that chemical formula was $\mathrm{Al}\left(\mathrm{SO}_{4}\right)_{3} \cdot 18 \mathrm{H}_{2} \mathrm{O}$ (Kento Chemical). The solutions with coagulant were rapid-mixed at $100 \mathrm{rpm}$ for $3 \mathrm{~min}$, slow-mixed at $30 \mathrm{rpm}$ for $15 \mathrm{~min}$, and allowed to settle for $20 \mathrm{~min}$. After completion of the settling process, supernatant samples were taken for measurement of turbidity via a turbidimeter (Hach).

Dissolved organic carbon (DOC), chlorine demand, THM, pH, and alkalinity analyses were performed for 
the treated water samples. The QA/QC programs set forth in Standard Methods (APHA, 1995) were followed for all sample analyses. Water samples for DOC and $\mathrm{UV}_{254}$ analyses were filtered through $0.45 \mu \mathrm{m}$ filters and determined by a TOC instrument (model 700, O.I. Corp.), and UV spectrophotometer (Hitachi U-2000) respectively. The chlorine concentration were adjusted to about 3 to $40 \mathrm{mgl}^{-1}$, which were depending on the chlorination period and would provide a free residual chlorine of at least 0.2 to $5 \mathrm{mgl}^{-1}$ at the end of the incubation period (APHA, 1995). The analysis of residual chlorine was performed by using the DPD $(N, N-$ diethyl- $p$-phenylene-diamine) ferrous titration method.

\section{Results and disscussion}

\subsection{Effects of polymers on coagulation enhancement at neutral ( $\mathrm{pH} 7$ ) condition}

Fig. 1 presents the results of jar test for water samples containing HA, TA and PHBA with alum coagulant at
$\mathrm{pH}$ 7. Under the neutralized condition, the concentration of flocs formed by $\mathrm{Al}(\mathrm{OH})_{3}$ was low and, therefore, flocs could not sweep the particles in water. Under acidic conditions, corrosion rate was accelerated that was not feasible in water treatment. They contribute to the concentration of suspension and resulted in high turbidity in alum-treated water (Adin et al., 1998). The dissolved organic carbon (DOC) in raw water was converted to a non-settling particulate form at low alum dosage and contributed to turbidity resulting in so-called "negative effect" phenomenon (White et al., 1997; Singer and Bilyk, 2002). Manahan (1994) found that the humic substances could bind the metal ions such as aluminum and iron. This binding can occur as chelation between a carboxyl group and a phenolic hydroxyl group. It was evidently shown in Fig. 1a that it required over $140 \mathrm{mgl}^{-1}$ of alum to render the residual turbidity lower than 2 NTU. Compared with the HA water, it requires less dosage of alum for treating the TA and PHBA water. At a specific alum dosage $\left(<140 \mathrm{mgl}^{-1}\right)$, the lower residual is associated with decreasing organic MW. The more complex structure and functional groups, the higher

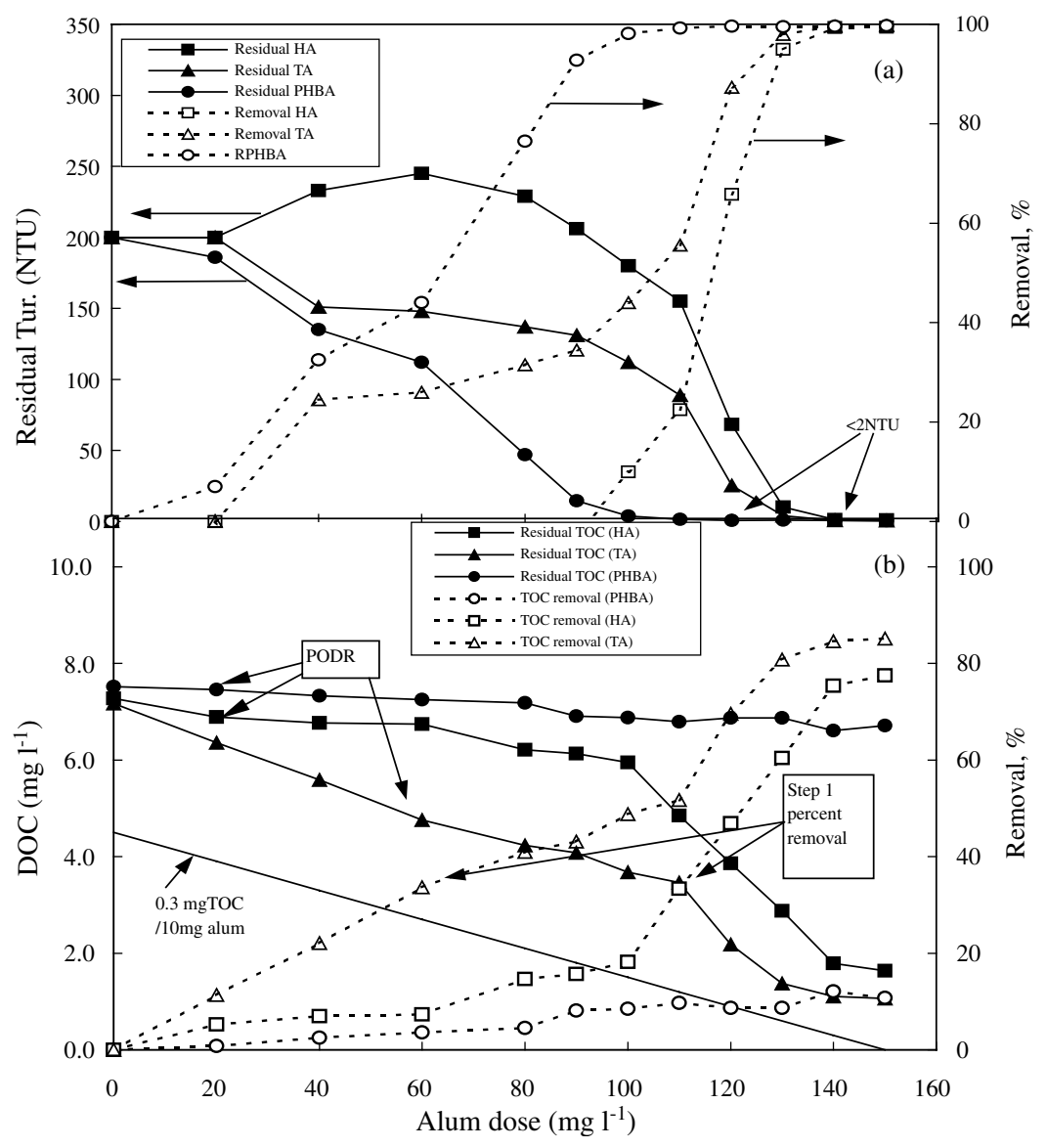

Fig. 1. Results of jar tests of (a) turbidity and (b) DOC for water samples by introducing alum dose at $\mathrm{pH}$ 7. (Raw water: DOC $=7.0 \pm 0.7 \mathrm{mgl}^{-1}$, Turbidity $=200 \pm 10 \mathrm{NTU}$, Alkalinity $=100 \pm 10 \mathrm{mgl}^{-1}$ as $\mathrm{CaCO}_{3}$.) 
chemical dosage is needed to destabilize the system (Divakaran and Pillai, 2001).

The requirement of DOC removal for enhanced coagulation suggested in the USEPA D/DBP Rules, provides an operational procedure to establish a point of diminishing returns (PODR) which is defined as the alum dosage beyond which $<0.3 \mathrm{mgl}^{-1}$ DOC is removed per $10 \mathrm{mgl}^{-1}$ addition of alum in various jar tests. $35 \%$ of DOC removal efficiency was set as an evaluation criterion in this research. It could be seen from Fig. 1b that the slope of DOC/alum became steeper over $100 \mathrm{mgl}^{-1}$ of alum, therefore, $100 \mathrm{mgl}^{-1}$ was the threshold dosage for HA water. It was obvious that TA was relatively easy to be removed by coagulation than HA. Alum has very little effects on PHBA removal at lower alum dosage, although it could remove the turbidity quite successfully.

Since great amounts of coagulants were needed to achieve DOC and turbidity removal requirements, various polymers are chosen as coagulant aids to enhance coagulation and reduce alum consumption. While treating HA water, the addition of CPAM could only reduce turbidity slightly. Non-ionic PAM had better efficiency in removing turbidity than CPAM (Table 1). It is because CPAM could neither adsorb positively charged flocs nor neutralize the charge of particles due to its low CD. Contrarily, using $p$-DADMAC as coagulantaid, the residual turbidity could be reduced to a lower level, even less than 1 NTU at higher dosages. For example, an alum dosage of $20 \mathrm{mgl}^{-1}, 80 \%$ of turbidity was removed with $8 \mathrm{mgl}^{-1}$ of $p$-DADMAC for both TA and HA. As alum dose increased, $p$-DADMAC addition could reduce turbidity significantly.

While treating PHBA water, both PAMs had better effects on enhancing the turbidity removal than treating
HA or TA water. Non-ionic PAM was still better than CPAM in turbidity removal, but even at the highest chemical dosage, $60 \mathrm{mgl}^{-1}$ of alum and $10 \mathrm{mgl}^{-1}$ of non-ionic PAM, the residual turbidity of treated water (41 NTU), was extremely higher than the Drinking Water Quality Standard in Taiwan. About $5 \mathrm{mgl}^{-1}$ of $p$-DADMAC does reduce the turbidity of treated water to lower than $2 \mathrm{NTU}$, regardless of the amount of alum dosage. It was evident that the addition of $p$-DADMAC had significant improvement on turbidity removal.

Among three types of polymers, $p$-DADMAC exhibits the most efficient performance for turbidity removal. Comparing the properties of polymers, both PAMs had higher molecular weight and lower CD than $p$-DADMAC. The difference in coagulation performances exhibited by the various type of polymer suggests that the $\mathrm{CD}$ of a polymer should be more influential than the molecular weight. In this investigation, it was found that the organic composition in water would affect the efficiency of turbidity removal. The organic compounds with complex structures and functional groups required higher chemical dosages to produce sufficient positive charged flocs for turbidity removal by charge neutralization and adsorption. It was thus concluded that the sequence of the amounts of chemical needed for turbidity removal be: HA > TA > PHBA.

Fig. 2 show DOC removal efficiencies for HA treated with polymers. While treating HA water, $p$-DADMAC is the only one to enhance the coagulation efficiency over the threshold of enhanced coagulation requirement over $35 \%$ of DOC removal. Both cationic and non-ionic PAM had little effects on DOC removal. Moreover, higher PAM dosage would remain in treated water and result in higher residual of DOC concentration.

Table 1

Residual turbidity in three types of NOMs water samples treated by alum plus polymer coagulation process ${ }^{\mathrm{a}}$

\begin{tabular}{|c|c|c|c|c|c|c|c|c|c|c|}
\hline \multicolumn{2}{|l|}{ Polymer } & \multicolumn{9}{|c|}{ Residual turbidity-NTU } \\
\hline \multirow[t]{2}{*}{ Type } & \multirow{2}{*}{$\begin{array}{l}\text { Dose level } \\
\left(\mathrm{mgl}^{-1}\right)\end{array}$} & \multicolumn{3}{|c|}{ Alum $20 \mathrm{mgl}^{-1}$} & \multicolumn{3}{|c|}{ Alum $40 \mathrm{mgl}^{-1}$} & \multicolumn{3}{|c|}{ Alum $60 \mathrm{mgl}^{-1}$} \\
\hline & & HA & TA & PHBA & HA & TA & PHBA & HA & TA & PHBA \\
\hline & & 200 & 162 & 186 & 236 & 130 & 167 & 262 & 117 & 113 \\
\hline$p$-DADMAC & 2 & 175 & 140 & 37.6 & 181 & 105 & 30.8 & 137 & 22.4 & 10.5 \\
\hline$p$-DADMAC & 5 & 103 & 98.0 & 0.9 & 38.3 & 7.6 & 1.8 & 11.2 & 1.2 & 1.7 \\
\hline$p$-DADMAC & 8 & 40.4 & 39.4 & 0.2 & 5.0 & 0.4 & 0.2 & 0.9 & 3.9 & 1.5 \\
\hline$p$-DADMAC & 10 & 3.8 & 14.0 & 0.7 & 0.6 & 0.5 & 0.6 & 0.4 & 3.3 & 4.9 \\
\hline $\operatorname{PAM}(+)$ & 2 & 185 & 159 & 197 & 213 & 130 & 177 & 220 & 114 & 98.3 \\
\hline $\operatorname{PAM}(+)$ & 5 & 174 & 144 & 182 & 206 & 120 & 142 & 204 & 104 & 86.1 \\
\hline $\operatorname{PAM}(+)$ & 8 & 166 & 147 & 165 & 197 & 118 & 134 & 183 & 104 & 81.2 \\
\hline $\operatorname{PAM}(+)$ & 10 & 168 & 161 & 153 & 182 & 116 & 119 & 174 & 106 & 70.0 \\
\hline PAM (non) & 2 & 171 & 131 & 171 & 205 & 127 & 140 & 218 & 113 & 74.5 \\
\hline AM (non) & 5 & 149 & 120 & 145 & 165 & 103 & 121 & 182 & 102 & 54.6 \\
\hline PAM (non) & 8 & 138 & 105 & 132 & 137 & 92.3 & 96.6 & 155 & 88.0 & 45.2 \\
\hline PAM (non) & 10 & 122 & 97.9 & 126 & 129 & 98.1 & 89.9 & 135 & 81.8 & 41.4 \\
\hline
\end{tabular}

${ }^{\mathrm{a}}$ Raw water: $\mathrm{DOC}=7.0 \pm 0.7 \mathrm{mgl}^{-1}$, turbidity $=200 \pm 3 \mathrm{NTU}$, alkalinity $=100 \pm 10 \mathrm{mgl}^{-1}$ as $\mathrm{CaCO}_{3}, \mathrm{pH}=7$. 


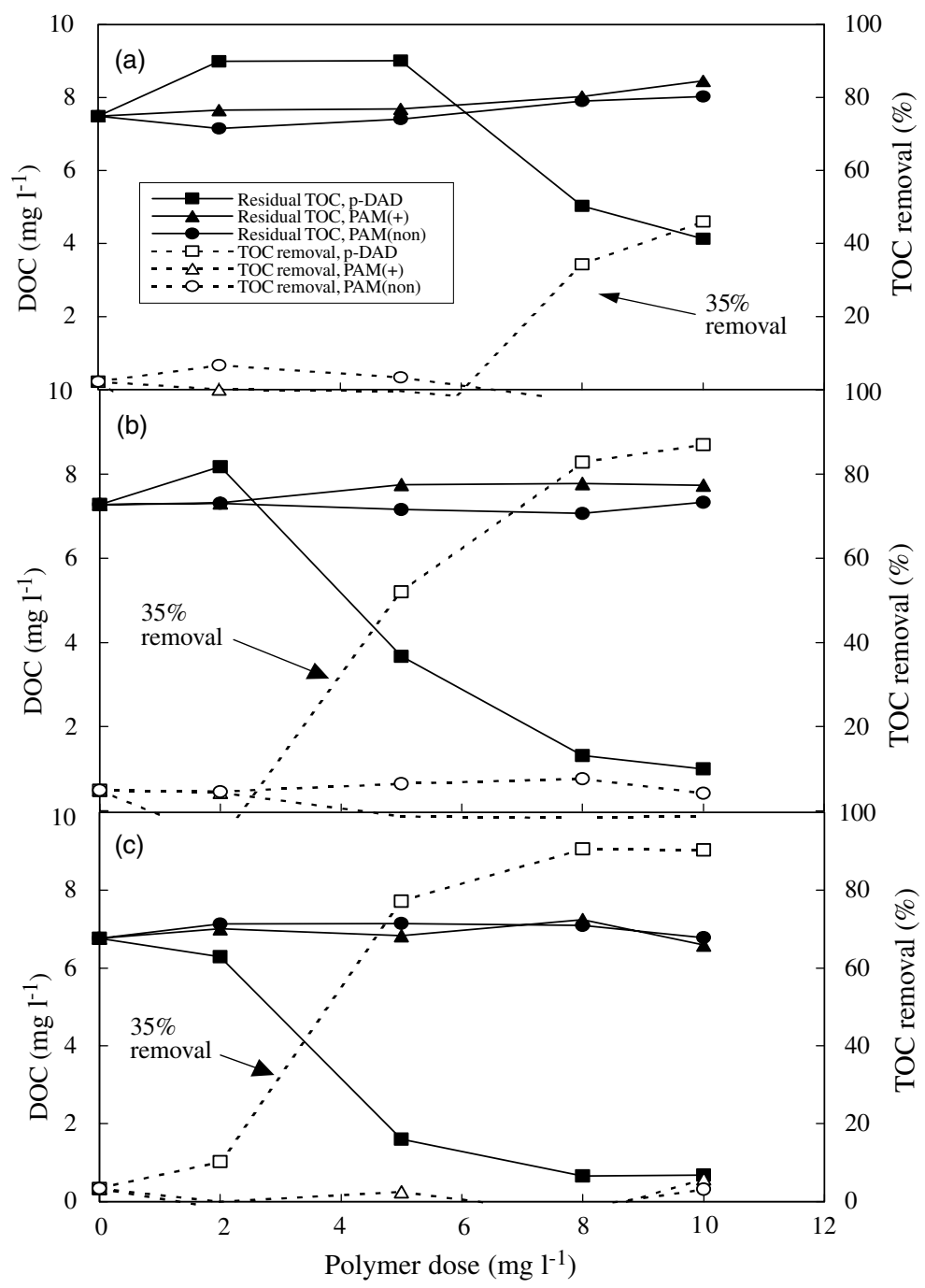

Fig. 2. Results of jar tests (DOC) for introducing alum as (a) Alum 20, (b) Alum 40, (c) Alum 60 plus polymers to humic acid water samples. (Raw water: DOC $=7.0 \pm 0.7 \mathrm{mgl}^{-1}$ as HA, Turbidity $=200 \pm 10 \mathrm{NTU}$, Alkalinity $=100 \pm 10 \mathrm{mgl}^{-1} \mathrm{as} \mathrm{CaCO}_{3}$.)

$p$-DADMAC could strengthen the linkage between particles and flocs, which enlarge the size of flocs and make them easier to settle. Furthermore, the organic matter might be adsorbed on to the flocs and be removed along with the precipitates. However, insufficient dosage of $p$ DADMAC would increase DOC concentration in treated water, and it was even much significant than the over-dosage of cationic and non-ionic PAM. While dosing $20 \mathrm{mgl}^{-1}$ of alum, over $8 \mathrm{mgl}^{-1}$ of $p$-DADMAC was needed to achieve the $35 \%$ DOC removal requirement. Similar patterns were observed that over $5 \mathrm{mgl}^{-1} p$ DADMAC was needed when $40 \mathrm{mgl}^{-1}$ of alum was added and $2 \mathrm{mgl}^{-1}$ of $p$-DADMAC with $60 \mathrm{mgl}^{-1}$ of alum. Thus, it can be concluded that the higher the alum dosage, the lower dosing $p$-DADMAC is needed for
DOC removal. Consequently, lower dose of $p$-DADMAC could easily link the particles and flocs together due to its high positive $\mathrm{CD}$ and results in the formation of polymer-floc complexes.

In general, results regarding DOC removals from the treatment of water containing TA were similar to those of HA water. Dosing $60 \mathrm{mgl}^{-1}$ of alum could reduce TOC concentration effectively, polymers might not be necessary unless higher removal requirement is needed. Both PAMs not only had little effects on DOC removal, but also impeded the coagulation performances regardless of changes in alum dosage. When the dosage of alum was $20 \mathrm{mgl}^{-1}$, more than $6 \mathrm{mgl}^{-1}$ of $p$-DADMAC was needed to achieve the enhanced coagulation requirement. 
Since alum alone was not capable of removing PHBA, different polymers were dosed to improve the coagulation performance. However, none of the polymers used in this research could enhance coagulation performance. It is evident that the hydrophilic property and smaller molecular weight of PHBA could impede the co-precipitation and adsorption of organic carbon resulted in lowering reduction of DOC.

\subsection{Effect of $p H$ adjustment on turbidity and DOC removal for $p-D A D M A C$}

The $\mathrm{pH}$ adjustment with metal salt coagulants is an important operating parameter for the coagulation process. Adjusting the $\mathrm{pH}$ to the range between 4 and 5 are generally believed to enhance the coagulation performance with alum. The $\mathrm{pH}$ of synthetic water was adjusted to 5, 6, and 7 prior to coagulant addition. While treating the HA water, $p$-DADMAC could help to remove most of the turbidity in water. At low alum dosage, e.g., $20 \mathrm{mgl}^{-1}$, the coagulation effects mainly were contributed by $p$-DADMAC, however, $\mathrm{pH}$ effect is not significant shown in Fig. 3a. When the alum dosage was increased to $60 \mathrm{mgl}^{-1}$, $\mathrm{pH}$ effects became obvious. As shown in Fig. 3b, the percent turbidity removal at $\mathrm{pH} 5$ is higher than that at $\mathrm{pH} 6$ or 7. The addition of $p$-DADMAC became useless at $\mathrm{pH} 5$ due to the sufficient alum and dosage. The role of $p$-DADMAC on treating TA water is similar to that on treating HA water. In general, the effects of $\mathrm{pH}$ became obvious with increasing alum dosage.

It was concluded that $\mathrm{pH}$ would affect the performance of alum in removing turbidity but not of $p$ DADMAC. Therefore, low $p$-DADMAC dosage in cooperation with high alum dosage would be affected by $\mathrm{pH}$ adjustment. Since a slight reverse of turbidity removal was observed at high polymer dosage and low $\mathrm{pH}$ for treating the above organic precursors, the dosage of polymer must be controlled well in low $\mathrm{pH}$ conditions.

In Fig. $3 c$ and d, it was observed that DOC removal increased with decreasing $\mathrm{pH}$ value at $20 \mathrm{mgl}^{-1}$ of alum dosage. It took about $5 \mathrm{mgl}^{-1}$ of $p$-DADMAC to achieve the same percent DOC removal requirement at $\mathrm{pH} 5$, while higher dosage was needed at higher $\mathrm{pH}$. (a) Alum $20 \mathrm{mgl}^{-1}$

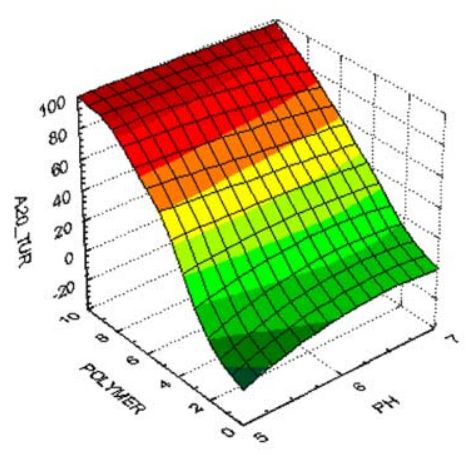

(c) Alum $20 \mathrm{mgl}^{-1}$

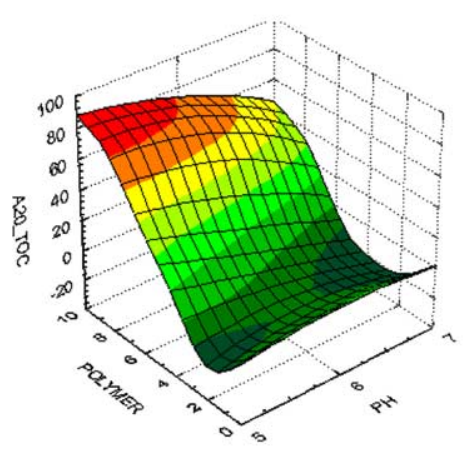

(b) Alum $60 \mathrm{mgl}^{-1}$

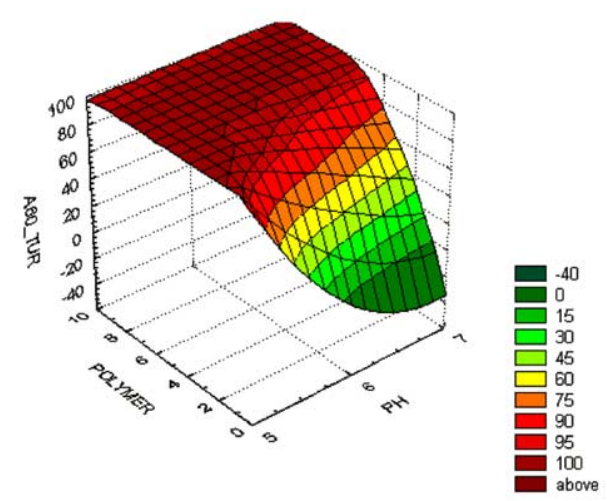

(d) Alum $60 \mathrm{mgl}^{-1}$

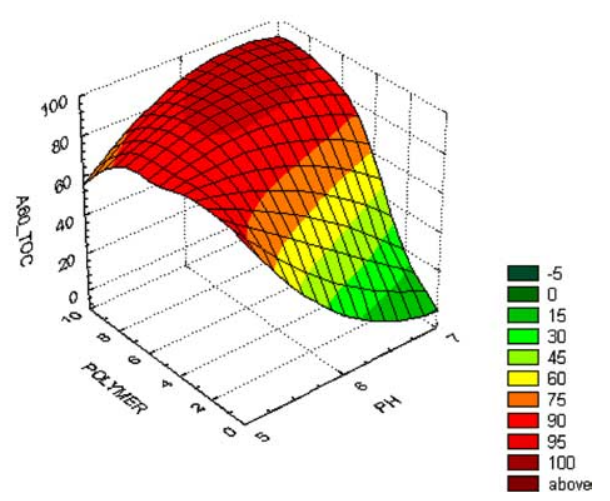

Fig. 3. Parameter removal (\%) as a function of $p$-DADMAC polymer dosage and $\mathrm{pH}$ for humic acid water. turbidity: (a) and (b); DOC: (c) and (d). 
However, when dosing $60 \mathrm{mgl}^{-1}$ of alum at $\mathrm{pH} 5$, higher dosage of $p$-DADMAC decreased DOC removal. High concentration of aluminum hydroxide species and hydrogen ions neutralized the negative charges on suspended particles during rapid mixing; part of the $p$ DADMAC added was utilized to bridge the particles. Therefore, excessive $p$-DADMAC dose would remain in water sample and contribute to DOC concentration. While treating the most irresponsive-to-coagulation organic matter, PHBA, pH adjustment combining polymer addition is ineffective, and there is no obvious relationship between chemical dosage and DOC removal efficiency.

Comparing the $U_{254}$ variation as shown in Fig. $4 a$, it is obvious that the concentration of organic matter with aromatic structure (HA in this case) decreased with increasing $p$-DADMAC dosage. In many studies, UV absorbance was used as a surrogate indicator for determining organic precursors (O'Melia et al., 1999; Singer and Bilyk, 2002). In this research, $\mathrm{UV}_{254}$ was also used as a supplementary index to determine the composition of organics in water. In order to determine the composition of organics in water treated by high polymer dose, $\mathrm{UV}_{254}$ is again used as an index, as shown in Fig. $4 \mathrm{~b}$. It can be observed that the reverse shows up when the concentration of DOC is already low in water treated by alum without polymer addition. It implies that if DOC is reduced to a low level, the addition of $p$-DADMAC must be controlled carefully for treating the water containing low level of DOC, otherwise, it might be useless and harmful.

\subsection{Reduction of THM formation potential (THMFP)}

Among three types of organic precursors, HA had the highest THM yield (110 $\mu \mathrm{g}$ THM/mg DOC), PHBA the second highest $(60 \mu \mathrm{g}$ THM/mg DOC) and TA the lowest at about $50 \mu \mathrm{g}$ THM/mg DOC. HA contains many activating functional groups such as hydroxyl, carbonyl, and acryloxy etc. which will react with chlorine to form THM.
Chlorine demand and THMFP are both related to DOC concentration. Enhanced coagulation by alum plus $p$-DADMAC could reduce the chlorine demand. The THMFP and chlorine demand of raw, alum-, and alum $p$-DADMAC-treated waters for three different synthetic waters are compared and showed in Fig. 5. For HA and TA water, much less reduction of chlorine demand were observed for the water treated. However, the higher reduction in chlorine demand and THMFP was only found in the HA and TA water treated with alum plus $p$-DADMAC.

The THMFP results of HA water and treated water were shown in Table 2. For the most cases of HA, THMFP was found to decrease with increasing chemical dosage. If eliminating the data that were not able to reduce the turbidities to a level lower than 2 NTU, coagulation treatment at $\mathrm{pH} 5$ could decrease THMFP by $85-94 \%$, and $89-96 \%$ at $\mathrm{pH} 7$. The results suggest that if strict standard (turbidity $<1 \mathrm{NTU}$ ) is adopted for treating high turbidity water in the future, high dosage of polymer is necessary to decrease THMFP effectively and, therefore, $\mathrm{pH}$ effect is not critical in this condition.

As the elimination based on residual turbidity standard, coagulation at $\mathrm{pH} 5$ could reduce $67-86 \%$ of

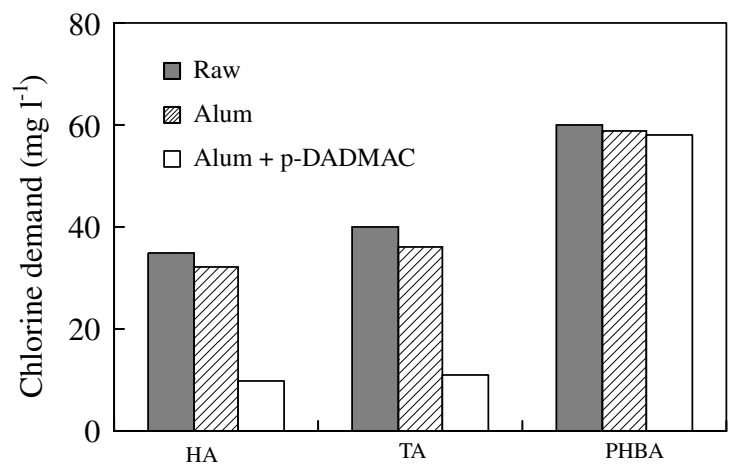

Fig. 5. Chlorine demand for raw, alum-, and alum $+p$-DADMAC-treated water for different source water $\left(7.0 \pm 0.7 \mathrm{mgl}^{-1}\right.$ DOC).

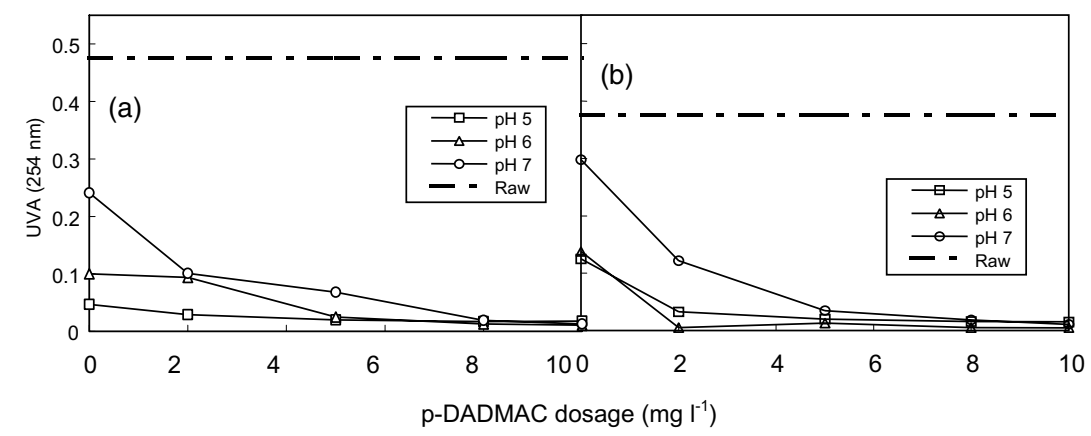

Fig. 4. $\mathrm{UV}_{254}$ (a) humic acid and (b) tannic acid as a function of $p$-DADMAC dosage at different $\mathrm{pH}$ (Alum dosage: $60 \mathrm{mg} 1^{-1}$ ). 
Table 2

Summary of THMFP percent removal in humic acid water samples treated by alum and alum plus $p$-DADMAC polymer

\begin{tabular}{|c|c|c|c|c|c|c|c|c|c|c|c|c|c|}
\hline \multicolumn{2}{|c|}{ Applied dosage } & \multicolumn{4}{|l|}{ pH 5} & \multicolumn{4}{|l|}{$\mathrm{pH} 6$} & \multicolumn{4}{|l|}{$\mathrm{pH} 7$} \\
\hline $\begin{array}{l}\text { Alum } \\
\left(\mathrm{mgl}^{-1}\right)\end{array}$ & $\begin{array}{l}p \text {-DADMAC } \\
\left(\mathrm{mgl}^{-1}\right)\end{array}$ & TOC $^{\mathrm{a}}$ & Tur $^{\mathrm{b}}$ & $\begin{array}{l}\text { THMFP } \\
\left(\mu \mathrm{g} 1^{-1}\right)\end{array}$ & $\begin{array}{l}\text { Percent } \\
\text { decrease }(\%)\end{array}$ & $\mathrm{TOC}^{\mathrm{a}}$ & Tur $^{\mathrm{b}}$ & $\begin{array}{l}\text { THMFP } \\
\left(\mu g 1^{-1}\right)\end{array}$ & $\begin{array}{l}\text { Percent } \\
\text { decrease (\%) }\end{array}$ & TOC $^{\mathrm{a}}$ & Tur $^{\mathrm{b}}$ & $\begin{array}{l}\text { THMFP } \\
\left(\mu \mathrm{gl}^{-1}\right)\end{array}$ & $\begin{array}{l}\text { Percent } \\
\text { decrease (\%) }\end{array}$ \\
\hline & & & & 1001 & & & & 846 & & & & 816 & \\
\hline 20 & 0 & + & + & 898 & 10 & + & + & 843 & 0 & + & + & 666 & 18 \\
\hline 20 & 2 & + & + & 784 & 22 & + & + & 848 & 0 & + & + & 846 & -4 \\
\hline 20 & 5 & + & + & 393 & 61 & + & + & 742 & 12 & + & + & 730 & 11 \\
\hline 20 & 8 & - & + & 69 & 93 & - & + & 284 & 66 & + & + & 278 & 66 \\
\hline 20 & 10 & - & - & 59 & 94 & - & - & 107 & 87 & - & - & 86 & 89 \\
\hline 40 & 0 & + & + & 900 & 10 & + & + & 769 & 9 & + & + & 540 & 34 \\
\hline 40 & 2 & + & + & 306 & 69 & + & + & 744 & 12 & + & + & 739 & 9 \\
\hline 40 & 5 & - & - & 70 & 93 & & + & 668 & 21 & - & + & 341 & 58 \\
\hline 40 & 8 & - & - & 53 & 95 & - & + & 83 & 90 & - & + & 76 & 91 \\
\hline 40 & 10 & - & - & 64 & 94 & - & - & 52 & 94 & - & - & 31 & 96 \\
\hline 60 & 0 & - & - & 145 & 85 & + & + & 582 & 31 & + & + & 654 & 20 \\
\hline 60 & 2 & - & - & 139 & 86 & - & + & 356 & 58 & + & + & 566 & 31 \\
\hline 60 & 5 & - & - & 113 & 89 & - & - & 124 & 85 & - & + & 85 & 90 \\
\hline 60 & 8 & - & - & 73 & 93 & - & - & 56 & 93 & - & - & 41 & 95 \\
\hline 60 & 10 & - & - & 75 & 92 & - & - & 54 & 94 & - & - & 60 & 93 \\
\hline
\end{tabular}

If percent TOC removal $>35 \%$; +: yes; - : no.

${ }^{\mathrm{b}}$ If residual turbidity $<2 \mathrm{NTU} ;+$ : yes; - : no. 
THMFP, $78-91 \%$ at $\mathrm{pH} 6$, and $83-90 \%$ at $\mathrm{pH}$ 7. Low percent THMFP reduction at $\mathrm{pH} 5$ is due to the poor performance of DOC removal caused by over-dosage of $p$-DADMAC. As a result, while treating TA water, higher $\mathrm{pH}$ will prevent over-dosage and lead to better THMFP reduction.

Fig. 6 further illustrated these relationships between THMFP and DOC for HA water and TA water. The linear relationship could be observed in both samples which was consistent with the findings suggested by other researchers (Page et al., 2002; Singer and Bilyk, 2002). The regression equations in Fig. 6a estimates that a $90 \mu \mathrm{gl}{ }^{-1}$ decrease in HM formation for every $1 \mathrm{mgl}^{-1}$ decrease in DOC concentration ( $90 \mu \mathrm{g}$ THM/mg DOC) and a $40 \mu \mathrm{g}$ THM $/ \mathrm{mg}$ DOC for HA and TA water, respectively, which are both higher than the result, $26 \mu \mathrm{g}$ THM/mg DOC, proposed by Singer and Bilyk (2002). It could be explained by that the selected organic precursors in this research are more generative in formation of THM than the raw water from the river of United States.

The distribution of THMFP at various polymer doses is illustrated in Fig. 7 which indicated that the median THMFP decrease and the range of the measured THMFP data narrow with increasing polymer dose. This observation suggests that high dosage of polymer, $p$-DADMAC, should possess great performance in removing DOC for treating HA and TA waters.

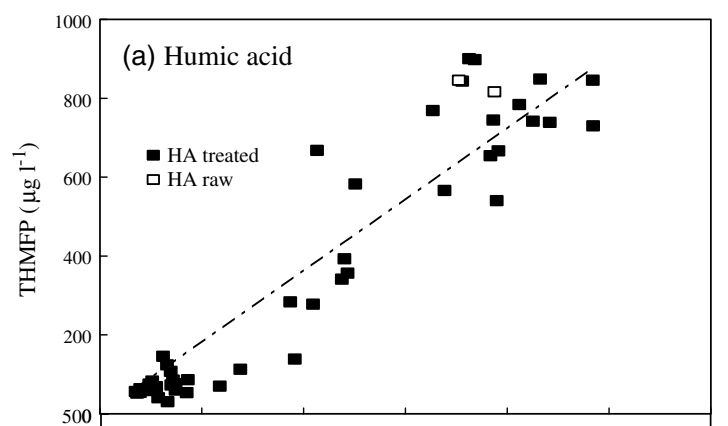

(b) Tannic acid

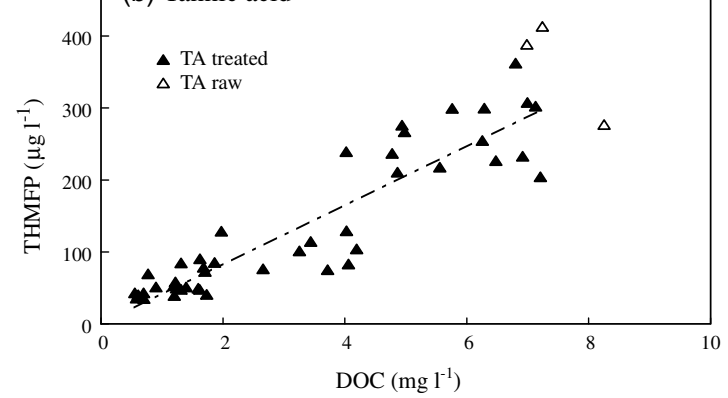

Fig. 6. Correlation between THMFP and DOC for (a) humic acid and (b) tannic acid.
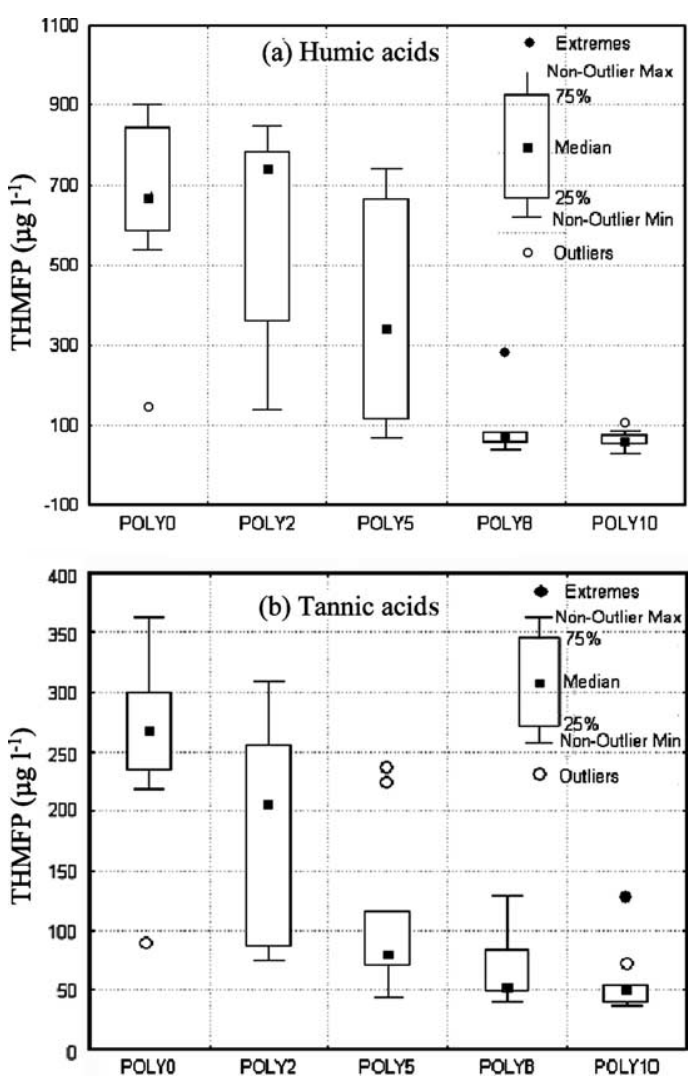

Fig. 7. Distribution of THMFP at various polymer doses (Alum dosage: $60 \mathrm{mgl}^{-1}$ ).

\section{Conclusions}

In this investigation, it was observed that at a specific $\mathrm{pH}(\mathrm{pH}=7)$ condition, the concentration of alum needed to decrease THMFP follows the order: HA > TA $>$ PHBA, and acidic condition is conducive to alum coagulation, and is more effective with increasing alum dosage. Enhanced coagulation with the addition of a polymer, $p$-DADMAC, was found to be very effective in removing high MW of THM precursors, such as HA and TA, from the synthetic waters, and markedly reduced the alum dosages required for turbidity removal. However, the removal of low MW of THM precursors, such as PHBA, was found to be unfavorable to coagulation.

Residual TDOC concentration, UV absorbance, turbidity, THM formation potential, and chlorine demand in HA and TA waters, except for PHBA waters, were all substantially lower as a result of adding $p$-DADMAC as coagulant-aid in alum coagulation. The other two polymers, cationic PAM and non-ionic PAM, which had higher molecular weight but lower charge density than $p$-DADMAC, were not capable of removing effectively any of the parameters mentioned above. The results 
implied that charge density was a very specific characteristic of polymer affecting the performance of coagulation process. While treating low turbidity water with $p$-DADMAC, its high charge density narrowed the optimum dosage and was impeditive to the performance of coagulation. As a result, using polymer to treat low turbidity water is not recommended.

\section{References}

Adin, A., Soffer, Y., Aim, R.B., 1998. Effluent pretreatment by iron coagulation applying various dose- $\mathrm{pH}$ combinations for optimum particle separation. Wat. Sci. Technol. 38, 27 34.

Anonymous, 1991. Final report on the assessment of polyacrylamide. J. Am. Coll. Toxicol. 10, 193-203.

APHA, 1995. Standard Methods for the examination of Water and Wastewater, 19th ed. American Public Health Association, Washington, DC.

AWWA, 1987. Standard for Poly(Diallydimethylammonium Chloride), AWWA B451-87. American Water Works Association, Denver, CO, USA.

Chang, E.E., Chaing, P.C., Chao, S.H., Liang, C.H., 1999. Effects of polydiallyldimethyl ammonium chloride coagulant on formation of chlorinated by products in drinking water. Chemosphere 39, 1333-1346.

Chang, E.E., Chiang, P.C., Ko, Y.W., Lan, W.H., 2001. Characteristics of organic precursors and their relationship with disinfection by-products. Chemosphere 44, 1231-1236.

Collins, M.R., Amy, G.L., Steelink, C., 1986. Molecular weight distribution, carboxylic acidity, and humic substances content of aquatic organic matter. Implications for removal during water treatment. Environ. Sci. Technol. 20, 1028 1032 .
Divakaran, R., Pillai, V.N.S., 2001. Flocculation of kaolinite suspensions in water by chitosan. Wat. Res. 35, 3904-3908.

Exell, K.N., Vanloon, G.W., 2000. Using coagulants to remove organic matter. J. Am. Water Works Assoc. 92, 93-102.

Mallevialle, J., Bruchet, A., Fiessinger, F., 1984. How safe are organic polymers in water treatment. J. Am Water Works Assoc. 76, 87-93.

Manahan, S.E., 1994. Environmental Chemistry. Lewis Publishers, Chelesea MI, pp. 80-81.

Mustafa, M.B., Walker, H.W., 2001. Effect of natural organic coatings on the polymer-induced coagulation of colloidal particles. Colloids Surf. A 177, 215-222.

O’Melia, C.R., Becker, W.C., Au, K.K., 1999. Removal of humic substances by coagulation. Wat. Sci. Technol. 40, 4754.

Owen, D.M., Amy, G.L., Chowdhury, Z.K., 1993. Characterization of Natural Organic Matter and its Relationship to Treatability. AWWA Res. Fund., Denver, CO, USA.

Page, D.W., van Leeuwen, J.A., Spark, K.M., Drikas, M., Withers, N., Mulcahy, D.E., 2002. Effect of alum treatment on the trihalomethane formation and bacterial regrowth potential of natural and synthetic waters. Wat. Res. 36, 4884-4892.

Ratnaweera, H., Gjessing, E., Oug, E., 1999. Influence of physical-chemical characteristics of natural organic matter (NOM) on coagulation properties: An analysis of eight Norwegian water sources. Wat. Sci. Technol. 40 (4-5), 8995.

Seybold, C.A., 1994. Polyacrylamide review: Soil conditioning and environmental fate. Commun. Soil Sci. Plant Anal. 25, 2171-2185.

Singer, P.C., Bilyk, K., 2002. Enhanced coagulation using a magnetic ion exchange resin. Wat. Res. 36, 4009-4022.

White, M.C., Thompson, J.D., Harrington, G.W., Singer, P.C., 1997. Evaluating criteria for enhanced coagulation compliance. J. Am. Water Works Assoc. 89, 64-77. 